This is a post-peer-review, pre-copyedited version of:

Calardo, Erica; Governatori, Guido; Rotolo, Antonino. Sequence Semantics for Modelling Reason-based Preferences, in «FUNDAMENTA INFORMATICAE», 2018, 158, pp. 217-238.

The final publication is available online at IOS Press through:

http://dx.doi.org/10.3233/FI-2018-1647 


\title{
Sequence Semantics for Modelling Reason-based Preferences
}

\author{
Erica Calardo
}

CIRSFID, University of Bologna

Via Galliera 3, 40122 Bologna, Italy

Guido Governatori

Data61, CSIRO

41 Boggo Road, Dutton Park, Queensland, 4109, Australia

\author{
Antonino Rotolo \\ CIRSFID, University of Bologna
}

Via Galliera 3, 40122 Bologna, Italy

\begin{abstract}
We study how the non-classical $n$-ary operator $\otimes$, originally intended to capture the concept of reparative obligation, can be used in the context of social choice theory to model preferences. A novel possible-world model-theoretic semantics, called sequence semantics, was proposed for the operator. In this paper, we propose a sound and complete axiomatisation of a minimal modal logic for the operator, and we extend it with axioms suitable to model social choice consistency principles such as extension consistency and contraction consistency. We provide completeness results for such extensions.
\end{abstract}

Keywords: Sequence semantics, preferences, modal logic, non normal modal logics, neighbourhood semantics, social choice theory, choice consistency

\section{Introduction}

Theoretical and computational research in social choice theory is now recognised as relevant and is well-established in the Multi-Agent System (MAS) community. Indeed, it deals with the problem of how to aggregate in MAS individual preferences into a social or collective preference in order to achieve a rational collective decision [1].

Address for correspondence: Guido Governatori, guido.governatori@data61.csiro.au 
Preliminarily to any useful contribution in this area we need to develop suitable formalisms and reasoning methods to represent and handle agents' preferences. In the current literature, we can find several approaches, among which the most remarkable in computational social choice theory are perhaps the following [2]:

- conditional preference networks, or CP-nets [3];

- prioritised goals $[4,5]$.

The second approach uses logical formalisms to describe the goals of the agents whose preferences are modelled as propositional formulae. This allows for a manageable and purely qualitative representation of preferences. Very recently, a new proposal in this perspective has been advanced [6], which presents a modal logic where a binary operator is meant to syntactically express preference orderings between formulae: Each formula of this logic determines a preference ordering over alternatives based on the priorities over properties that the formula express. Accordingly, such types of formalisms are in fact capable of representing not just orderings over alternatives but the reasons that lead to the preferences [7]. The formalism is then interestingly used in [6] to originally treat the problem of collective choice in MAS as aggregation of logical formulae. The logic in [6] is clearly inspired by the work in [8], which in turn has a number of similarities with a system that was independently developed in [9] and where a Gentzen system was proposed in a different but related area—deontic logic—-to reason about orderings on obligations. The idea that reasoning about preferences is crucial in deontic logic was introduced in semantic settings long time ago [10] (for recent discussions see [11, 12]). However, [9] is based on the syntactic introduction of the new non-classical operator $\otimes$ : The reading of an expression like $a \otimes b \otimes c$ is that $a$ is the primary obligation, but if this obligation is violated, the secondary obligation is $b$, and, if the secondary (contrary-to-duty) obligation $b$ is violated as well, then $c$ is obligatory. These constructions can be used as well to reason about preferences. Thus, following the approach in [9], an expression like

$$
\text { Resident } \rightarrow \neg \text { Pay_Taxes } \otimes \neg \text { Pay_Interest } \otimes \text { Pay_Minimum }
$$

can be intuitively viewed as a conditional preference statement meaning the following:

1. if I'm resident in Italy, i.e., if Resident is the case, then not paying taxes is my actual preference, but,

2. if it happens that I pay taxes, then my actual preference is rather not to pay any interest, but

3. if I pay any interest, then my actual preference is pay a minimum.

The advantage of this formalism is that we can offer a compact representation of reasons explaining preferences, and thus we can also develop intuitive logical tools for reasoning about individual choices. As it was proposed in the tradition of deontic logic (see [11, 12]) and as it has been recently recalled by [6] in the context choice theory, modal logics are a natural way of modelling reason-based preferences. Indeed, preferences among propositional formulae correspond semantically to ideality or orderings on possible worlds. Very recently, we have devised a new semantics for $\otimes$ deontic logics, which extends neighbourhood models with sequences of truth sets $[13,14]$. In this paper we extend our previous work and offer new results for a novel preference logic. More precisely, we address in this paper the following research questions: 
- What is the minimal axiomatisation for modelling reason-based preferences using $\otimes$ ? We will see that the minimal system $\mathrm{E}^{\otimes}$ just extends classical modal systems [15] even though some additional schemata can be proposed to grasp reasonable properties of preferences.

- How to semantically characterise the idea of reason-based preference? We will argue that the intuition of $[13,14]$ can be applied to preference logic: the idea is that an expression like $a \otimes b$ is semantically reflected in the sequence $\left\langle\|a\|_{V},\|b\|_{V}\right\rangle$ of truth sets. Specific soundness and completeness results will be proved for preference logics.

- How to relate the formalism with well-known properties of preferences studied in rational choice theory? We will discuss how to express two basic social choice principles (contraction and expansion) in the $\otimes$-logic.

The layout of the paper is as follows. After the introduction of the language used (Section 2), Section 3 presents the basic logical system for $\otimes$ to represent and reason about preferences. The logic recalls some intuitions from $[9,13,14]$. Section 4 defines a sequence neighbourhood semantics suitable for the system which adjusts the one proposed in $[13,14]$. Sections 5 and 6 provide soundness and completeness results. In Section 7 we discuss how to apply the logic to the modelling of some social choice principles (i.e., contraction and expansion). Some conclusions end the paper.

\section{Language}

The language consists of a countable set of atomic formulae. Well-formed-formulae are then defined using the usual Boolean connectives and the $n$-ary connective $\otimes$, which is intended to syntactically formalise a preference ordering among reasons. The language also includes the modal operator Pr denoting the actual preferred reason or state of affairs: in other words, $\operatorname{Pr} p$ means that $p$ is actually preferred.

The interpretation of an expression $a \otimes b$ is that $a$ is the most preferred reason or state of affairs, but, if $a$ is not the case then $b$ is preferred.

Let $\mathcal{L}$ be a language consisting of a countable set of propositional letters Prop $=\left\{p_{1}, p_{2}, \ldots\right\}$, the propositional constant $\perp$, round brackets, the Boolean connective $\rightarrow$, the unary operator $\operatorname{Pr}$, and a set of $n$-ary operators $\otimes^{n}$ for $n \in \mathbb{N}, n>0$. We will refer to the $\otimes^{n}$ operators and $\operatorname{Pr}$ as preference operators.

The main motivation for having a family of $\otimes$ operators instead of a single binary operator plus commutativity, i.e., $a \otimes(b \otimes c) \equiv(a \otimes b) \otimes c$, is to prevent nested preference expressions. With this option one would conflate preferences and "meta-preferences" in the same reading, and it will establish preferences over formulae representing concepts of different types, e.g., $(a \otimes b)$ and $c$. Furthermore, as we have alluded to above, given the expression (or as we will call it $\otimes$-chain) $A \otimes c$ we form the preference $c$, if $A$ does not hold. But, if $A=(a \otimes b)$, what does it mean that it does not hold. Is it that $a$ is not preferred to $b$ or there is no preference between $a$ and $b$, or $a$ is preferred to $\neg b$. While there are no particular technical problems to adapt the development to a binary operator, we refrain to do that until we have a clear conceptual reading of nested $\otimes$-chain expressions.

\section{Definition 2.1. (Well Formed Formulae)}

Well formed formulae (wffs) are defined as follows:

- Any propositional letter $p \in \operatorname{Prop}$ and $\perp$ are wffs; 
- If $a$ and $b$ are wffs, then $a \rightarrow b$ is a wff;

- If $a$ is a wff and no preference operator occurs in $a$, then $\operatorname{Pr} a$ is a wff;

- If $a_{1}, \ldots, a_{n}$ are wffs and no preference operator occurs in any of the $a_{i}, 1 \leq i \leq n$, then $a_{1} \otimes^{n} \cdots \otimes^{n} a_{n}$ is a wff, where $1 \leq n ;{ }^{1}$

- Nothing else is a wff.

We use WFF to denote the set of well formed formulae.

Other Boolean operators are defined in the standard way, in particular $\neg a={ }_{\text {def }} a \rightarrow \perp$ and $\top=_{\text {def }}$ $\perp \rightarrow \perp$.

We say that any formula $a_{1} \otimes^{n} \cdots \otimes^{n} a_{n}$ is an $\otimes$-chain of length $n$; also the negation of an $\otimes$-chain is an $\otimes$-chain. The formation rules allow us to have $\otimes$-chain of any (finite) length, and the arity of the operator is equal to number of elements in the chain; accordingly, we drop the index $m$ from $\otimes^{m}$. Moreover, we will often use the prefix notation $\bigotimes_{i=j}^{n} a_{i}$ for $a_{j} \otimes \cdots \otimes a_{n}$; however, since the arity of the operator can be deduced by the number of arguments, we shall henceforth drop the index. In addition we use the following notation: $\bigotimes_{i=j}^{n} a_{i} \otimes b \otimes \bigotimes_{k=l}^{m} c_{k}$, where $j, l \in\{0,1\}$. The " $a$ " part and " $c$ " part are optional, i.e., they are empty when $i=0$ or $k=0$, respectively. Otherwise the expression stands for the following chain of $n+1+m$ elements: $a_{1} \otimes \cdots \otimes a_{n} \otimes b \otimes c_{1} \otimes \cdots \otimes c_{m}$.

\section{Axioms}

In this section we are going to examine which basic axioms can be chosen for a logic intended to model ordered preferences. We assume that any such logic is based on a suitable axiomatisation of classical propositional logic.

\subsection{Minimal System $E^{\otimes}$}

The first axiom we consider is the following:

$$
\left.\bigotimes_{i=1}^{n} a_{i} \equiv\left(\bigotimes_{i=1}^{k-1} a_{i}\right) \otimes\left(\bigotimes_{i=k+1}^{n} a_{i}\right) \quad\left(\text { where } a_{j} \equiv a_{k}, \text { for some } j<k\right) \quad \text { ( } \otimes \text {-shortening }\right)
$$

This axiom allows us to remove (introduce) duplicate formulae (or equivalent formulae) from the left. Thus, given the $\otimes$-chain

$$
a \otimes b \otimes a \otimes c
$$

we can derive the equivalent formula

$$
a \otimes b \otimes c
$$

The intuition is as follows: As we have seen in Section 1 the meaning of $\otimes$-chain in (2) is that $a$ is the most preferred option, but if that is not possible, then the formula following it, in this case $b$, is the next preferred option, and so on. Specifically, if $b$ does not hold, then $a$ is the new preferred option, but we

\footnotetext{
${ }^{1}$ We will use the prefix form $\otimes{ }^{1} a$ for the case of $n=1$.
} 
already know that $a$ does not hold, and then we can move to the successive option, i.e., $c$. Intuitively, if I prefer not to get any damage, but if this happens I prefer to be compensated, and, if the damage is not compensated, then I prefer not to get any damage, this just means that my primary preference is not to get any damage and my secondary preference is to be compensated.

Notice that Axiom $\otimes$-shortening gives us a method to define a normal form for preferences: Given any $\otimes$-chain expression, we can create the shortest $\otimes$-chain where all the elements in it are disjoint. We are going to use this property for the development of the semantics for the logic of $\otimes$-chains (see Definitions 4.2, 4.3 and 4.5 below).

The next axiom allows us to derive actual preferences (i.e., formualae in the scope of the modal preference operator $\operatorname{Pr}$ from $\otimes$-chains). Thus it provides a form of detachment for deriving actual preferences from $\otimes$-chains, i.e., those preferences that hold in a given context. They reflect the intuitive reading of the $\otimes$ operator. Indeed, if $a \otimes b$, the primary preference should hold, and, if $a$ is factually false $(\neg a)$, then $b$ must be preferred, i.e., $\operatorname{Pr} b:^{2}$

$$
\left(\left(\left(\bigotimes_{i=0}^{n} b_{i}\right) \otimes c \otimes\left(\bigotimes_{j=0}^{m} d_{j}\right)\right) \wedge\left(\bigwedge_{i=0}^{n} \neg b_{i}\right)\right) \rightarrow \operatorname{Pr} c \quad \text { (Pr-detachment) }
$$

Notice that when the initial optional prefix is absent, Axiom Pr-detachment can be rewritten as

$$
\bigotimes_{i=1}^{n} a_{i} \rightarrow \operatorname{Pr} a_{1}
$$

Thus, the first element of an $\otimes$-chain is one of our actual preferences.

Let us illustrate how the axiom works with the help on an example. Consider the formula (1) where I reside in Italy and I have paid my taxes. This means that Resident holds, from which, by modus ponens, we obtain

$$
\neg \text { Pay_Taxes } \otimes \neg \text { Pay_Interest } \otimes \text { Pay_Minimum }
$$

and then by Pr-detachment (or Pr-detachment-1) we can conclude Pr $\neg P a y_{-}$Taxes. If I am a good citizen, and I pay my taxes (against my preference), i.e., Pay_Taxes holds, then Pr-detachment allows me to infer the not paying interest is now one of my preferences, that is we derive $\operatorname{Pr} \neg P a y \_$Interest. Finally, the minimal system $\mathrm{E}^{\otimes}$ is equipped with the following two inference rules, that permit replacement of equivalent formulae in the context of $\otimes$ and Pr.

$$
\begin{gathered}
\frac{\bigwedge_{i=1}^{n}\left(a_{i} \equiv b_{i}\right)}{\left(\bigotimes_{i=1}^{n} a_{i}\right) \equiv\left(\bigotimes_{i=1}^{n} b_{i}\right)} \\
\frac{a \equiv b}{\operatorname{Pr} a \equiv \operatorname{Pr} b}
\end{gathered}
$$

\subsection{Additional Axioms}

In Section 4 we are going to introduce a novel possible world sequence semantics for $\otimes$ and $\operatorname{Pr}$, and in Section 5 we are going to show that the logic $\mathrm{E}^{\otimes}$ is the minimal system for the semantics. The minimal

\footnotetext{
${ }^{2}$ Please, remember the convention that if the subscript starts with 0 , then that part of the sequence is optional.
} 
logic does not allow us to determine whether the preferences of an agent are consistent, and it does not provide mechanisms to derive (new) preferences from existing ones. In this section we present some additional axioms establishing consistency principles for preferences, and a mechanism to generate new preferences from existing ones. These axioms can be used to extend the minimal logic ${ }^{\otimes}$. In Section 7 we are going to examine what axioms are needed to represent some social choice principles.

We begin with an axiom $(\otimes-I)$ that allows us to derive new preference orderings by combining existing preference orderings (thus it corresponds to a peculiar introduction rule for $\otimes$ ).

$$
\left(\left(\bigotimes_{k=0}^{p} a_{k} \otimes \bigotimes_{i=1}^{n} b_{i} \otimes \bigotimes_{l=0}^{q} c_{l}\right) \wedge\left(\bigwedge_{i=1}^{n} \neg b_{i} \rightarrow \bigotimes_{j=1}^{m} d_{j}\right)\right) \rightarrow \bigotimes_{k=0}^{p} a_{k} \otimes \bigotimes_{i=1}^{n} b_{i} \otimes \bigotimes_{j=1}^{m} d_{j}
$$

Let us illustrate $(\otimes-\mathrm{I})$ by considering a simple instance of it as applied to a concrete example:

$$
\begin{gathered}
\neg \text { Pay_Taxes } \otimes \neg \text { Pay_Interest } \\
\text { Pay_Taxes } \wedge \text { Pay_Interest } \rightarrow \otimes \otimes^{1} \text { Pay_Minimum }
\end{gathered}
$$

The formula in (4) states that my primary preference is not to pay taxes, but if this happens then my preference is not pay any interest (for example, by paying them in due time without delay). The expression in (5) specifies that, if I pay taxes and pay with interest (e.g., because I was late), then my preference is to pay the minimum amount. Hence, $(\otimes-I)$ states that there is a chain of preferences dealing iteratively with the fact that my primary preference (not to pay any taxes) is not satisfied.

The next two axioms can be use to guarantee that preferences are consistent.

$$
\operatorname{Pr} a \rightarrow \neg \operatorname{Pr} \neg a
$$

Axiom Pr-D is the standard D axiom of modal logic [15] and it represents the principle of internal consistency of actual preferences. Thus, when the axiom is assumed, it is not possible to have that an agent prefers at the same time $a$ and its opposite $\neg a$. "External consistency" of preferences is given

$$
\bigotimes_{i=1}^{n} a_{i} \rightarrow \bigotimes_{i=1}^{n-1} a_{i}
$$

Clearly, given that we use classical propositional logic as the underlying logic, it is not possible that an $\otimes$-chain and its negation hold at the same time. However, without axiom $\otimes-\perp$, it is possible to have that $\otimes$-chains like $a \otimes b \otimes c$ and $\neg(a \otimes b)$ hold. The first $\otimes$-chain states that $a$ is preferred, the second best preference is $b$, and the third best preferred one is $c$. But $\neg(a \otimes b)$ assert that $b$ is not the second best preference with respect to $a$. This case is subsumed by $a \otimes b \otimes c$, thus $a \otimes b \otimes c$ and $\neg(a \otimes b)$ (or $\neg \otimes^{1} a$ ) should result in a contradiction. Thus axiom $\otimes-\perp$ ensures this effect by establishing that if an agent subscribes to a particular preference ordering, then the agent subscribes to (derives) all the initial (starting from the leftmost element) sub-orderings (sub-chains) of an existing $\otimes$-chain. For example, if

$$
\neg \text { Pay_Taxes } \otimes \neg \text { Pay_Interest } \otimes \text { Pay_Minimum }
$$

holds, then we can conclude that the following hold, too:

$$
\begin{gathered}
\neg \text { Pay_Taxes } \otimes \neg \text { Pay_Interest } \\
\otimes^{1} \neg \text { Pay_Taxes. }
\end{gathered}
$$




\section{Sequence Semantics}

In this section we are going to introduce a novel semantics, called sequence semantics, for the logic of $\otimes$-chains. The motivation for the development of such a semantics is twofolds. First, the semantics is a generalisation of standard possible worlds neighbourhood semantics for modal logic. Thus, this new setting can incorporate existing work where preferences are modelled using modal operators. Second, the semantics allows us to capture the behaviour of $\otimes$-chains. Sequence semantics was first proposed in the context of deontic logic to obviate the problem that, in some cases, what constitutes the violation of a compensated obligation can be evaluated as permitted [16]. Consider the $\otimes$-chain $a \otimes b$ : in terms of possible worlds this expression means that we prefer a possible world where $a$ holds to a world where $a$ does not hold; furthermore, we prefer a world where both $\neg a$ and $b$ holds to one where $\neg a$ holds but $b$ does not; and the world where $\neg a \wedge b$ holds still respects the preference given by $a \otimes b$. Thus, an $\otimes$-chain induces an order on possible worlds. Accordingly, the induced order can be use as the accessibility relation for Pr. Suppose that for some reasons $\neg a$ holds everywhere in the model, thus the first element of the order over the possible world is where $\neg a \wedge b$ holds. Using now the standard evaluation clause for modalities in Kripke models, we get $\operatorname{Pr} \neg a$, but this does not correspond to the preference encoded by $a \otimes b$.

Let us introduce the semantic structures that we use to interpret $\otimes$-formulae. In fact, they are just an extension of neighbourhood frames for classical modal logics.

Definition 4.1. A sequence frame is a tuple $\mathcal{F}=\langle W, \mathcal{C}\rangle$ where:

- $W$ is a non empty set of worlds;

- $\mathcal{C}$ is a neighbourhood function with the following signature ${ }^{3}$

$$
\mathcal{C}: W \rightarrow 2^{\left(\left(2^{W}\right)^{n}\right)} \quad \text { for } n \in \mathbb{N} .
$$

In general, a sequence frame is nothing but a structure where the standard neighbourhood function is replaced by a function that establishes an order between elements (i.e., sets of worlds) of each neighbourhood associated to every world. Notice that in case of $n=1$ the structure is isomorphic to a standard neighbourhood frame. Figure 1 offers a pictorial representation of the intuition.

The following definitions introduce the notion of redundancy and the operations of zipping and $s$-zipping, i.e., operations that, respectively, remove repetitions or redundancies occurring in $\otimes$-chains and in sequences of sets of worlds. Intuitively, these operations are necessary because, despite the fact the language allows for building expressions like $a \otimes b \otimes a$, these must be semantically evaluated using the sequence of sets of worlds $\left\langle\|a\|_{V},\|b\|_{V}\right\rangle$ (see rule ( $\otimes$-shortening)).

Definition 4.2. A formula $A$ is redundant iff $A=\bigotimes_{i=1}^{n} a_{i}, n>1$ and $\exists a_{j}, a_{k}, 1 \leq j, k \leq n, j \neq k$, such that $a_{j} \equiv a_{k}$.

Definition 4.3. Let $A=\bigotimes_{i=1}^{n} a_{i}$ be any redundant formula. We say that the non-redundant $B$ is zipped from $A$ iff $B$ is obtained from $A$ by applying recursively the operations below:

\footnotetext{
${ }^{3}$ As done sometimes with the standard neighbourhood function, we use the notation $\mathcal{C}_{w}$ to denote $\mathcal{C}(w)$.
} 


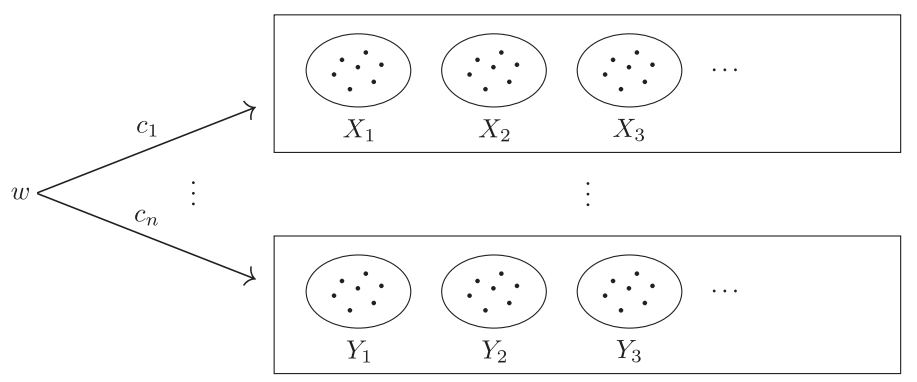

Figure 1: Basic sequence structure: $X_{1}, X_{2}, X_{3}, \cdots \subseteq W$ and $Y_{1}, Y_{2}, Y_{3}, \cdots \subseteq W$

1. If $n=2$, i.e., $A=a_{1} \otimes a_{2}$, and $a_{1} \equiv a_{2}$, then $B$, its zipped form, is $\operatorname{Pr} a_{1}$;

2. Otherwise, if $n>2$, then for $1 \leq k \leq n$, if $a_{j} \equiv a_{k}$, for $j<k$, delete $\otimes a_{k}$ from the sequence.

Let $X=\left\langle X_{1}, \ldots, X_{n}\right\rangle$ be such that $X_{i} \in 2^{W}(1 \leq i \leq n)$. We analogously say that $Y$ is s-zipped from $X$ iff $Y$ is obtained from $X$ by applying the operations below:

1. If $n=2$ and $X_{1}=X_{2}$, then its s-zipped from $Y$ is $\left\langle X_{1}\right\rangle$;

2. Otherwise, if $n>2$, then for $1 \leq k \leq n$, if $X_{j}=X_{k}$, for $j<k$, delete $X_{k}$ from the sequence.

Proposition 4.4. Let $A$ and $B$ be two formulae such as $B$ is zipped from $A$, then $A \equiv B$.

\section{Proof:}

The proof trivially follows from the definition of zipped from and axiom $\otimes$-shortening.

Given two formulas $A$ and $B$ where $A$ is not redundant and $A$ is zipped from $B$ we will also say that $A$ is the zipped form of $B$.

\section{Definition 4.5. (Models with sequences and truth of formulae)}

A model $\mathcal{M}$ is a pair $\langle\mathcal{F}, V\rangle$ where $\mathcal{F}$ is a sequence frame and $V$ is a valuation such that:

- for any non-redundant formula $\bigotimes_{i=1}^{n} a_{i}, \models_{w}^{V} \bigotimes_{i=1}^{n} a_{i}$ iff there is a $c_{j} \in \mathcal{C}_{w}$ such that $c_{j}=$ $\left\langle\left\|a_{1}\right\|_{V}, \ldots,\left\|a_{n}\right\|_{V}\right\rangle$

- for any redundant formula $\bigotimes_{i=1}^{n} a_{i}, \models_{w}^{V} \bigotimes_{i=1}^{n} a_{i}$ iff

- $\bigotimes_{f=1}^{k} a_{f}$ is zipped from $\bigotimes_{i=1}^{n} a_{i}$, and

$-\models_{w}^{V} \otimes_{f=1}^{k} a_{f}$.

- $\models{ }_{w}^{V} \operatorname{Pr} a$ iff there is a $c_{l} \in \mathcal{C}_{w}$ such that:

$-c_{l}=\left\langle\left\|a_{1}\right\|_{V}, \ldots,\left\|a_{n}\right\|_{V}\right\rangle$ 
- for some $k \leq n,\left\|a_{k}\right\|_{V}=\|a\|_{V}$;

- for $1 \leq j<k, w \notin\left\|a_{j}\right\|_{V}$.

Figure 2 pictorially illustrates the type of models used for evaluating $\otimes$-chains. In fact, we use only finite sequences of sets of worlds, closed under s-zipping. A formula $\bigotimes_{i=1}^{n} a_{i}$ is true iff the corresponding appropriate finite sequence of sets of worlds (without redundancies) is in $\mathcal{C}_{w}$. Notice that the evaluation clause for $\operatorname{Pr} a$ works using sequences of length 1 or with longer sequences whenever $a$ is the $k$ 's element of the $\otimes$-chain and the previous $a_{j}$ are such that $w \notin\left\|a_{j}\right\|_{V}$, i.e., the previous preferences have not been satisfied in $w$.

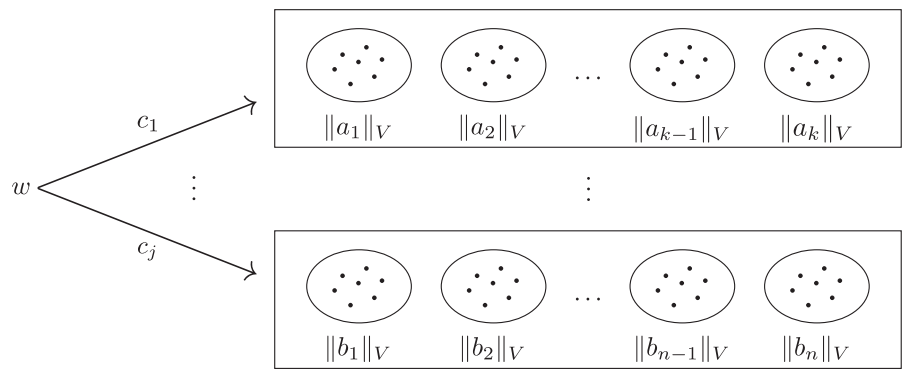

Figure 2: Sequence models where finite sequences are used to evaluate the formulae $\bigotimes_{i=1}^{k} a_{i}, \ldots, \bigotimes_{i=1}^{n} b_{i}$

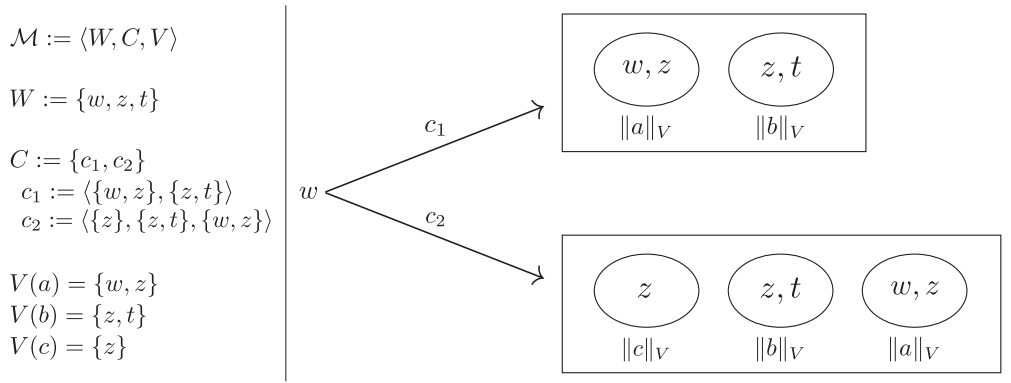

Figure 3: Example of a simple sequence model

The evaluation of $\otimes$-formulae is exemplified in Figure 3. Let $\mathcal{M}=\langle W, \mathcal{C}, V\rangle$ be a model where $W=\{w, z, t\}, \mathcal{C}_{w}=\left\{c_{1}, c_{2}\right\}, c_{1}=\langle\{w, z\},\{z, t\}\rangle, c_{2}=\langle\{z\},\{z, t\},\{w, z\}\rangle, V(a)=\{w, z\}$, $V(b)=\{z, t\}$, and $V(c)=\{z\}$. Given the valuation $V$, it holds that $c_{1}=\left\langle\|a\|_{V},\|b\|_{V}\right\rangle$ and hence the formula $a \otimes b$ is true at $w$, i.e., $\models{ }_{w}^{V} a \otimes b$; the same holds for the formula $c \otimes b \otimes a$, due to $c_{2}$. On the other hand $\forall \psi_{w}^{V} a \otimes b \otimes c$, since there is no sequence $\left\langle\|a\|_{V},\|b\|_{V},\|c\|_{V}\right\rangle$ associated to the world $w$. Finally, the formula $\operatorname{Pr} b$ is also satisfied; indeed $c_{2}=\left\langle\|c\|_{V},\|b\|_{V},\|a\|_{V}\right\rangle$ and $w \notin\|c\|_{V}$. 


\section{Soundness Results}

We now prove that the rules and axioms of system $E^{\otimes}$, namely rules $(\otimes$-shortening), (Pr-detachment), $(\otimes-$ $\mathrm{RE})$ and $(\operatorname{Pr}-\mathrm{RE})$, and schemata $(\otimes-\mathrm{I}),(\operatorname{Pr}-\mathrm{D})$ and $(\otimes-\perp)$ are sound.

Lemma 5.1. $\otimes$-RE, Pr-RE, $\otimes$-contraction, Pr-detachment are valid in the class of all sequence frames.

\section{Proof:}

The result for $(\otimes-\mathrm{RE})$ and (Pr-RE) trivially follows from the fact that the valuation clause for any $\otimes$-chain $\bigotimes_{i=1}^{n} a_{i}$, at any world $w$ and with any valuation $V$, requires the existence of a sequence $c \in \mathcal{C}_{w}$ of truth sets $\left\langle\left\|a_{1}\right\|_{V}, \ldots,\left\|a_{n}\right\|_{V}\right\rangle$. Then since for any $i,\left\|a_{i}\right\|_{V}=\left\|b_{i}\right\|_{V}\left(a_{i} \equiv b_{i}\right.$ for any frame and any valuation by assumption) there is also a sequence $\left\langle\left\|b_{1}\right\|_{V}, \ldots,\left\|b_{n}\right\|_{V}\right\rangle \in \mathcal{C}_{w}$.

The proof for $\otimes$-contraction follows directly from the valuation clause of redundant formulae, and from the definitions of redundancy, zipping, and s-zipping.

The proof for Pr-detachment follows from the valuation clause for the operator Pr.

\section{Definition 5.2. (Extended Frames)}

A sequence frame is extended when the following holds. For any world $w$ if there is a sequence $\left\langle A_{0}, \ldots, A_{i}, B_{1}, \ldots, B_{k}, C_{0}, \ldots, C_{j}\right\rangle \in \mathcal{C}_{w}$ such that $w \notin B_{l}$ for $1 \leq l \leq k$ implies $\left\langle D_{1}, \ldots, D_{m}\right\rangle \in$ $\mathcal{C}_{w}$, then $\left\langle A_{0}, \ldots, A_{i}, B_{1}, \ldots, B_{k}, D_{1}, \ldots, D_{m}\right\rangle \in \mathcal{C}_{w}$

\section{Definition 5.3. (Serial Frames)}

A sequence frame is serial if the following holds. Given any world $w$, if $\left\langle A_{0}, \ldots, A_{i}, C_{1}, \ldots, C_{k}\right\rangle \in \mathcal{C}_{w}$ and $w \notin A_{l}$ for $0 \leq l \leq i$, then for any sequence $\left\langle D_{0}, \ldots, D_{m}\right\rangle$ such that for $0 \leq l \leq m, w \notin D_{l}$, it holds that $\left\langle D_{0}, \ldots, D_{m}, W-C_{1}\right\rangle \notin \mathcal{C}_{w}$.

\section{Definition 5.4. $(\otimes-$-Serial Frames)}

A sequence frame is $\otimes$-serial iff for any world $w$, if $\left\langle A_{1}, \ldots, A_{n}\right\rangle \in \mathcal{C}_{w}$ and $n \geq 2$, then $\left\langle A_{1}, \ldots, A_{n-1}\right\rangle \in$ $\mathcal{C}_{w}$.

\section{Lemma 5.5.}

1. Schema $(\otimes-\mathrm{I})$ is valid in the class of extended frames.

2. Schema (Pr-D), is valid in the class of serial frames.

3. Schema $(\otimes-\perp)$ is valid in the class of $\otimes$-serial frames.

\section{Proof:}

The proof for all three cases is easy and straightforward following the syntactic structure of the corresponding axioms. 


\section{Completeness Results}

Before introducing the definition of canonical sequence model, some technical definitions are required. First, it should be explained what it means for two $\otimes$-chains to be consistent.

Proposition 6.1. Let $A$ and $B$ be two $\otimes$-chains. $A$ and $B$ are inconsistent (i.e., $A \wedge B \rightarrow \perp$ ) iff

- $A=\bigotimes_{i=1}^{n} a_{i}, B=\neg\left(\bigotimes_{j=1}^{m} b_{j}\right)$,

- $C=\bigotimes_{j=1}^{k} c_{j}$ is zipped from $A$,

- $D=\bigotimes_{j=1}^{k} d_{j}$ is zipped from $\bigotimes_{j=1}^{m} b_{j}$, and

- for all $1 \leq l \leq k, c_{l} \equiv d_{l}$.

Proof:

1. $c_{1} \otimes \cdots \otimes c_{k} \equiv c_{1} \otimes \cdots \otimes c_{k} \quad$ tautology

2. $c_{1} \otimes \cdots \otimes c_{k} \equiv d_{1} \otimes \cdots \otimes d_{k} \quad 1, \otimes-\mathrm{RE}$

3. $\quad c_{1} \otimes \cdots \otimes c_{k} \equiv a_{1} \otimes \cdots \otimes a_{n} \quad \otimes$-shortening $C$ is zipped from $A$

4. $\quad a_{1} \otimes \cdots \otimes a_{n} \equiv d_{1} \otimes \cdots \otimes d_{k} \quad 2,3, \mathrm{PC}$

5. $b_{1} \otimes \cdots \otimes b_{m} \equiv d_{1} \otimes \cdots \otimes d_{k} \quad \otimes$-shortening $D$ is zipped from $B$

6. $\quad a_{1} \otimes \cdots \otimes a_{n} \equiv b_{1} \otimes \cdots \otimes b_{m} \quad 4,5, \mathrm{PC}$

7. $\quad a_{1} \otimes \cdots \otimes a_{n} \wedge \neg\left(b_{1} \otimes \cdots \otimes b_{m}\right) \rightarrow \perp \quad 6, \mathrm{PC}$

\section{Definition 6.2. ( $\otimes$-maximality)}

A set of formulae $w$ is $\otimes$-maximal if and only if it is (classically) maximal and for any $\otimes$-chain $\bigotimes_{i=1}^{n} a_{i}$, either $\bigotimes_{i=1}^{n} a_{i} \in w$, or $\neg \bigotimes_{i=1}^{n} a_{i} \in w$.

Given any maximal consistent set $w, w$ can be extended to a $\otimes$-maximal consistent set $w^{+}$by adding, for any $\bigotimes_{i=1}^{n} a_{i}$, either it, or its negation (preserving consistency) to $w$ in a standard recursive procedure.

\section{Lemma 6.3. (Lindenbaum's Lemma)}

Let $\mathrm{S}$ be any theory extending $\mathrm{E}^{\otimes}$. Any consistent set $w$ of formulae in the language $\mathcal{L}$ can be extended to a consistent $\mathcal{L}$-maximal set $w^{+}$.

\section{Proof:}

Let $a_{1}, a_{2}, \ldots$ be an enumeration of all the possible formulae in $\mathcal{L}$.

- $w_{0}:=w$;

- $w_{n+1}:=w_{n} \cup\left\{a_{n}\right\}$ if its closure under the axioms and rules of $\mathbf{S}$ is consistent, $w \cup\left\{\neg a_{n}\right\}$ otherwise;

- $w^{+}:=\bigcup_{n \geq 0} w_{n}$. 
The construction of a sequence canonical model is as follows.

Definition 6.4. $\left(E^{\otimes}\right.$-Canonical Models)

A sequence model $\mathcal{M}:=\left\langle W^{+}, \mathcal{C}, V\right\rangle$ is a canonical sequence model for $\mathrm{E}^{\otimes}$ if and only if:

1. $W^{+}$is the set of all the $\otimes$-maximal consistent sets.

2. For any propositional letter $p \in \operatorname{Prop},\|p\|_{V}:=|p|_{\mathrm{E}^{\otimes}}$, where $|p|_{\mathrm{E}^{\otimes}}:=\left\{w \in W^{+} \mid p \in w\right\}$.

3. Let $\mathcal{C}:=\bigcup_{w \in W^{+}} \mathcal{C}_{w}$, where for each $w \in W^{+}$,

$$
\mathcal{C}_{w}:=\left\{\left\langle\left\|a_{1}\right\|_{V}, \ldots,\left\|a_{n}\right\|_{V}\right\rangle \mid \bigotimes_{i=1}^{n} a_{i} \in w\right\} \cup\left\{\left\langle\|a\|_{V}\right\rangle \mid \operatorname{Pr} a \in w\right\},
$$

where each $a_{i}$ is a meta-variable for a Boolean formula.

\section{Lemma 6.5. (Truth Lemma)}

Given a canonical sequence model $\mathcal{M}$, for any $w \in W^{+}$and for any formula $A, A \in w$ if and only if $\models{ }_{w}^{V} A$.

\section{Proof:}

Given the construction of the canonical model, this proof is easy and can be given by induction on the length of an expression $A$. We consider only a few relevant cases.

Assume $A$ has the form $a_{1} \otimes \cdots \otimes a_{n}$ and is redundant (clearly the case for non redundant formulae is easier and does not need to be considered here). Suppose $a_{i} \otimes \cdots \otimes a_{n} \in w$. Then, by $\otimes$-shortening, we have that the formula $b_{1} \otimes \cdots \otimes b_{j}$, the zipped form of $A$, is also in $w$. By definition of canonical model we have that there is a sequence $\left\langle\left\|b_{1}\right\|_{V}, \ldots,\left\|b_{j}\right\|_{V}\right\rangle \in \mathcal{C}_{w}$. Following from the semantic clauses given to evaluate $\otimes$-chains, it holds that $\models_{w}^{V} a_{1} \otimes \cdots \otimes a_{n}$.

Now suppose that $\models_{w}^{V} a_{1} \otimes \cdots \otimes a_{n}$. By definition, there is a zipped formula $b_{1} \otimes \cdots \otimes b_{j}$ such that $\models_{w}^{V} b_{1} \otimes \cdots \otimes b_{j}$. Thus, $C_{w}$ contains an ordered $j$-tuple $\left\langle\left\|b_{1}\right\|_{V}, \ldots,\left\|b_{j}\right\|_{V}\right\rangle$. By definition of $\mathcal{C}_{w}$ it follows that $b_{1} \otimes \cdots \otimes b_{j} \in w$ and by $\otimes$-shortening, all the unzipped forms of $b_{1} \otimes \cdots \otimes b_{j}$ are also in $w$, including $a_{1} \otimes \cdots \otimes a_{n}$.

If $A$ has the form $\operatorname{Pr} b$ and $\operatorname{Pr} b \in w$, then $\left\langle\|b\|_{V}\right\rangle \in \mathcal{C}_{w}$ and, by definition $\models_{w}^{V} \operatorname{Pr} b$. Conversely, if $\models_{w}^{V} \operatorname{Pr} b$, then there is an s-zipped sequence $\left\langle\left\|c_{0}\right\|_{V}, \ldots,\left\|c_{n}\right\|_{V},\|b\|_{V},\left\|d_{1}\right\|_{V}, \ldots,\left\|d_{m}\right\|_{V}\right\rangle \in \mathcal{C}_{w}$ and for $0 \leq i \leq n, w \notin\left\|c_{i}\right\|_{V}$. Thus, since any $c_{i}$ is Boolean and $w$ is maximal, $\neg c_{0}, \ldots, \neg c_{n} \in w$. Moreover $\bigotimes_{i=0}^{n} c_{i} \otimes b \otimes \bigotimes_{j=1}^{m} d_{j} \in w$. Hence by the Pr-detachment rule, $\operatorname{Pr} b \in w$.

It is easy to verify that the canonical model exists and it is a sequence semantics model. For the non vacuity of $W^{+}$one can take a maximal consistent set of Boolean formulae and extend it with all non negated $\otimes$-formulae.

Consider any formula $A \notin \mathrm{E}^{\otimes} ;\{\neg A\}$ is consistent and it can be extended to a maximal set $w$ such that for some canonical model, $w \in W^{+}$. By Lemma 6.5, $w \nvdash_{V} A$.

Corollary 6.6. The system $\mathrm{E}^{\otimes}$ is sound and complete with respect to the class of sequence frames.

We shall now present some further results for systems obtained by adding additional schemata to our basic logic. The major difference with the case of the basic logic is that in the Lindenbaum's construction we have to be consistent with the logics obtained from $\mathrm{E}^{\otimes}$ extended with the relevant axioms. 


\section{Theorem 6.7.}

1. The system $\mathrm{E}^{\otimes} \oplus(\otimes-\mathrm{I})$ is sound and complete w.r.t. the class of extended frames.

2. The system $E^{\otimes} \oplus(\otimes-\mathbf{D})$ is sound and complete w.r.t. the class of serial frames.

3. The system $\mathrm{E}^{\otimes} \oplus(\otimes-\perp)$ is sound and complete w.r.t. the class of $\otimes$-serial frames.

Proof:

1. Take a world $w \in W^{+}$and a sequence $\left\langle\left\|\left.a_{0}\right|_{V}, \ldots,\right\| a_{i}\left\|_{V},\right\| b_{1}\left\|_{V}, \ldots,\right\| b_{k}\left\|_{V},\right\| c_{1}\left\|_{V}, \ldots,\right\| c_{j} \|_{V}\right\rangle$ $\in \mathcal{C}_{w}$ such that if $w \notin\left\|b_{l}\right\|_{V}$ for $1 \leq l \leq k$ then $\left\langle\left\|d_{1}\right\|_{V}, \ldots,\left\|d_{m}\right\|_{V}\right\rangle \in \mathcal{C}_{w}$. By construction it follows that (a) $\bigotimes_{n=0}^{i} a_{n} \otimes \bigotimes_{n=1}^{k} b_{n} \otimes \bigotimes_{n=0}^{j} c_{n} \in w$, and (b) $\bigwedge_{n=1}^{k} \neg b_{n} \in w$ implies $\bigotimes_{n=1}^{m} d_{n} \in$ $w$ and hence $\bigwedge_{n=1}^{k} \neg b_{n} \rightarrow \bigotimes_{n=1}^{m} d_{n} \in w$. Applying schema $(\otimes-\mathrm{I})$ and modus ponens, $\bigotimes_{n=0}^{i} a_{n} \otimes$ $\bigotimes_{n=1}^{k} b_{n} \otimes \bigotimes_{n=0}^{m} d_{n} \in w$, hence $\left\langle\left\|a_{0}\right\|_{V}, \ldots,\left\|a_{i}\right\|_{V},\left\|b_{1}\right\|_{V}, \ldots,\left\|b_{k}\right\|_{V},\left\|d_{1}\right\|_{V}, \ldots,\left\|d_{m}\right\|_{V}\right\rangle \in$ $\mathcal{C}_{w}$.

2. Take a world $w \in W^{+}$and a sequence $\left\langle\left\|a_{0}\right\|_{V}, \ldots,\left\|a_{i}\right\|_{V},\left\|c_{1}\right\|_{V}, \ldots,\left\|c_{k}\right\|_{V}\right\rangle \in \mathcal{C}_{w}$ such that $w \notin\left\|a_{l}\right\|_{V}$ for $0 \leq l \leq i$. Thus $\bigwedge_{n=1}^{i} \neg a_{n} \in w$. By Axiom (Pr-detachment) and modus ponens it follows that $\operatorname{Pr} c_{1} \in w$ and, by Axiom (Pr-D), $\neg \operatorname{Pr} \neg c_{1} \in w$. Assume by reductio that $\left\langle\left\|d_{0}\right\|_{V}, \ldots,\left\|d_{m}\right\|_{V}, W^{+}-\left\|c_{1}\right\|_{V}\right\rangle \in \mathcal{C}_{w}$ where $w \notin\left\|d_{l}\right\|_{V}$ for $0 \leq l \leq m$. Then $\bigotimes_{n=0}^{m} d_{n} \otimes$ $\neg c_{1} \in w$; again, by Axiom (Pr-detachment) and modus ponens, $\operatorname{Pr} \neg c_{1} \in w$ thus reaching a contradiction.

3. This is quite straightforward. If for some $w$ in the canonical model $\left\langle\left\|a_{1}\right\|_{V}, \ldots,\left\|a_{n}\right\|_{V}\right\rangle \in \mathcal{C}_{w}$ and $2 \leq n$, then $\bigotimes_{1}^{n} a_{i} \in w$ and, by Axiom $(\otimes-\perp)$ and modus ponens, $\bigotimes_{1}^{n-1} a_{i} \in w$, implying that $\left\langle\left\|a_{1}\right\|_{V}, \ldots,\left\|a_{n-1}\right\|_{V}\right\rangle \in \mathcal{C}_{w}$.

Corollary 6.8. The system $E^{\otimes} \oplus(\otimes-I) \oplus(\operatorname{Pr}-\mathbf{D}) \oplus(\otimes-\perp)$ is sound and complete w.r.t. the the class of extended, serial, and $\otimes$-serial frames.

\section{Choice Consistency: Contraction and Expansion}

It is almost standard in social choice theory to assume two rationality conditions of choice (which are related with the fact that a choice function is rationalisable) [1]: contraction consistency and expansion consistency. The former one "is concerned with keeping a chosen alternative choosable as the set is expanded by adding alternatives dominated [...] in other choices", while the latter one "is concerned with keeping a chosen alternative choosable as the set is contracted by dropping other alternatives" [17, page 65]. More precisely, contraction consistency states that if an agent chooses some alternative from a set $S$ of alternatives and such alternative remains available in a subset $S^{\prime}$ of $S$, then the agent chooses it from $S^{\prime}$. Expansion consistency somehow works in the opposite direction and requires that, given two sets $S$ and $S^{\prime}$ of alternatives such that $S \subseteq S^{\prime}$, for all pairs of alternatives in $S$, if one agent chooses two alternatives from $S$, then the agent still chooses both of them from $S^{\prime}$, or does not choose any of them [1]. Although it has been argued that in order to avoid Arrow's impossibility one possibility, among others, is 
precisely to relax one of the aforementioned principles [1,2], these two principles are usually taken as basic standards of rationality in choice theory.

We believe that there are at least two possible ways to interpret the above social choice principles in our framework. The first option is to consider the sets of options $S$ and $S^{\prime}$ as description of the situations. In such case contraction consistency can be represented by the classical concatenation:

$$
\frac{a \rightarrow b \quad b \rightarrow \bigotimes_{i=1}^{n} c_{i}}{a \rightarrow \bigotimes_{i=1}^{n} c_{i}}
$$

The basic idea is that $b$ is a set of conditions under which the preferences expressed by the $\otimes$-chain $\bigotimes_{i=1}^{n} c_{i}$ can be formed, and $a \rightarrow b$ means that in every state where $a$ holds $b$ holds as well. Thus, by monotonicity and transitivity of classical propositional logic, we can infer that we can form the preference $\bigotimes_{i=1}^{n} c_{i}$ given $a$.

Similarly, expansion consistency can be modelled in classical propositional logic as

$$
\frac{a \rightarrow \bigotimes_{i=1}^{n} b_{i} \quad c \rightarrow \bigotimes_{i=1}^{n} b_{i}}{\left((a \vee d) \rightarrow \bigotimes_{i=1}^{n} b_{i}\right) \equiv\left((c \vee d) \rightarrow \bigotimes_{i=1}^{n} b_{i}\right)}
$$

Here, pairs of alternatives (more generally, pairs of sets of alternatives) are selected by assuming the truth of $a$ and $c$ and we state that a certain choice from $\bigotimes_{i=1}^{n} b_{i}$ is considered in both alternatives. Now, if pick up larger sets (determined by disjunctively adding any arbitrary propositional formula $d$ ), then either the same choice is preserved, or it is abandoned in both alternatives.

The second alternative, that we are going to examine in details in the remainder of the current section, is to provide a modal characterisation of the two principles, similarly to what was proposed in the framework of [6], where a simple semantic formulation is advanced, but no syntactic formalisation is given. Our logic can satisfy both conditions under some additional frame conditions, and a simple formalisation is possible.

\subsection{Contraction}

Let us begin by considering contraction, which is exemplified as follows.

Example 7.1. Let $G=\{$ Erica, Serena $\}$ be the group of girls in a class $T=G \cup B$ consisting of boys and girls, where $B=\{$ Guido, Nino $\}$. If I prefer Serena (it is for me the best element) as the fastest runner over $100 \mathrm{~m}$ in the whole class, then I prefer Serena as also the fastest runner in $G$.

Within our formalism, choices are ordered via the $\otimes$ operator, while a simple way to select arbitrary sets of alternatives is done by arbitrarily considering propositional formulae. Hence, if we consider Example 7.1, contraction can be easily represented as follows ${ }^{4}$ :

$$
\frac{\text { SerenaFastest- } \mathrm{T} \rightarrow \text { SerenaFastest-G }}{(\text { SerenaFastest-T } \otimes \text { EricaFastest-T }) \rightarrow(\text { SerenaFastest-G } \otimes \text { EricaFastest- } \mathrm{T})}
$$

Contraction is clearly a generalisation of RM, i.e., the closure of $\otimes$ under logical implication:

\footnotetext{
${ }^{4}$ Indeed, let us state that SerenaFastest-T $=_{\text {def }}$ SerenaFastest-B $\wedge$ SerenaFastest-G.
} 


$$
\frac{\bigwedge_{i=1}^{n}\left(a_{i} \rightarrow b_{i}\right)}{\left(\bigotimes_{i=1}^{m} a_{i}\right) \rightarrow\left(\bigotimes_{i=1}^{m} b_{i}\right)}
$$

$(\otimes-\mathrm{RM} / \otimes-$ expansion $)$

This inference rule is not valid in general in sequence semantics, but rather in a subclass defined by the following property.

Definition 7.2. Let $\mathcal{F}=\langle W, \mathcal{C}\rangle$ be a sequence frame. We say that $\mathcal{F}$ is $\otimes$-supplemented iff, for any $w \in W$, if $\left\langle Y_{1} \cap Z_{1}, \ldots, Y_{m} \cap Z_{m}\right\rangle \in \mathcal{C}_{w}$, then $\left\langle Y_{1}, \ldots, Y_{m}\right\rangle \in \mathcal{C}_{w}$ and $\left\langle Z_{1}, \ldots, Z_{m}\right\rangle \in \mathcal{C}_{w}$.

This frame property validates schema $\otimes-\mathrm{M}$ below, which is the generalisation of the standard axiom schema M [15]:

$$
\left(\bigotimes_{k=1}^{m}\left(b_{k} \wedge c_{k}\right)\right) \rightarrow\left(\left(\bigotimes_{k=1}^{m} b_{k}\right) \wedge\left(\bigotimes_{k=1}^{m} c_{k}\right)\right)
$$

Lemma 7.3. $(\otimes-\mathbf{M})$ is valid in the class of $\otimes$-supplemented sequence frames.

\section{Proof:}

Suppose by reductio that there is an $\otimes$-supplemented frame $\mathcal{F}$, a valuation $V$, and a world $v$ such that

$$
\begin{gathered}
\models_{v}^{V} \bigotimes_{k=1}^{m}\left(b_{k} \wedge c_{k}\right) \\
\forall_{v}^{V} \bigotimes_{k=1}^{m} b_{k} .
\end{gathered}
$$

From (7) we obtain that there is a sequence $\left\langle\left\|b_{1}\right\|_{V} \cap\left\|c_{1}\right\|_{V}, \ldots,\left\|b_{m}\right\|_{V} \cap\left\|c_{m}\right\|_{V}\right\rangle \in \mathcal{C}_{v}$. From (8) we have that there is no sequence $\left\langle\left\|b_{1}\right\|_{V}, \ldots,\left\|b_{m}\right\|_{V}\right\rangle \in \mathcal{C}_{v}$; thus, we have that $\mathcal{F}$ is not $\otimes$-supplemented, contrary to our assumption.

Notice that $\otimes$-supplementation is canonical, thus completeness is ensured accordingly.

\section{Theorem 7.4. $\left(\right.$ Completeness of $\mathrm{EM}^{\otimes}$ )}

$E \mathrm{M}^{\otimes}$ is complete with respect to the class of sequence frames that are $\otimes$-supplemented.

\section{Proof:}

Let us consider a canonical model for $(\otimes-\mathbf{M})$. Let us consider any sequence $\left\langle\left\|b_{1}\right\|_{V} \cap\left\|c_{1}\right\|_{V}, \ldots,\left\|b_{m}\right\|_{V} \cap\right.$ $\left.\left\|c_{m}\right\|_{V}\right\rangle \in \mathcal{C}_{w}$. Clearly, $\left\langle\left\|b_{1} \wedge c_{1}\right\|_{V}, \ldots,\left\|b_{m} \wedge c_{m}\right\|_{V}\right\rangle \in \mathcal{C}_{w}$. Then, $\left(\bigotimes_{k=1}^{m}\left(b_{k} \wedge c_{k}\right)\right) \in w$. Since $(\otimes-\mathbf{M})$ is valid (Lemma 7.3), then $\left(\bigotimes_{k=1}^{m} b_{k} \wedge \bigotimes_{k=1}^{m} c_{k}\right) \in w$. Hence, $\left(\bigotimes_{k=1}^{m} b_{k}\right) \in w$ and $\left(\bigotimes_{k=1}^{m} c_{k}\right) \in w$. By construction, $\left\langle\left\|b_{1}\right\|_{V}, \ldots,\left\|b_{m}\right\|_{V}\right\rangle \in \mathcal{C}_{w}$ and $\left\langle\left\|c_{1}\right\|_{V}, \ldots,\left\|c_{m}\right\|_{V}\right\rangle \in \mathcal{C}_{w}$. Therefore, the model is $\otimes$-supplemented.

The following result shows that the logic $\mathrm{EM}^{\otimes}$ equals to $\mathrm{E}^{\otimes}$ plus the rule $\otimes$-RM, thus $\otimes$-supplementation characterises $\otimes-\mathrm{RM} /$ contraction.

Lemma 7.5. The logic $E M^{\otimes}$ equals the logic $\mathrm{E}^{\otimes}$ plus the rule $\otimes-\mathrm{RM}$. 


\section{Proof:}

The proof follows easily if we can show that $\otimes-\mathrm{RM}$ is a derived rule in $\mathrm{EM}^{\otimes}$ and $\otimes-\mathrm{M}$ can be derived in the logic $E^{\otimes}$ plus the rule $\otimes-R M$.

We first show that $\otimes-\mathrm{RM}$ can be derived in $\mathrm{EM}^{\otimes}$.

$$
\begin{array}{lll}
\text { 1. } & \bigwedge_{i=1}^{n}\left(a_{i} \rightarrow b_{i}\right) & \text { Assumption } \\
\text { 2. } & \bigwedge_{i=1}^{n}\left(a_{i} \equiv a_{i} \wedge b_{i}\right) & \text { 1., PC } \\
\text { 3. } & \left(\bigotimes_{i=1}^{n} a_{i}\right) \equiv\left(\bigotimes_{i=1}^{n} a_{i} \wedge b_{i}\right) & \text { 2., } \otimes-\mathrm{RE} \\
\text { 4. } & \left.\left(\bigotimes_{i=1}^{n} a_{i} \wedge b_{i}\right)\right) \rightarrow\left(\left(\bigotimes_{i=1}^{n} a_{i}\right) \wedge\left(\bigotimes_{i=1}^{n} b_{i}\right)\right) & \text { Instance of } \otimes-\mathrm{M} \\
\text { 5. } & \left(\bigotimes_{i=1}^{n} a_{i}\right) \rightarrow\left(\left(\bigotimes_{i=1}^{n} a_{i}\right) \wedge\left(\bigotimes_{i=1}^{n} b_{i}\right)\right) & \text { 3., 4., PC } \\
\text { 6. } & \left(\bigotimes_{i=1}^{n} a_{i}\right) \rightarrow\left(\bigotimes_{i=1}^{n} b_{i}\right) & \text { 5., PC }
\end{array}
$$

Let us now prove that $\otimes-\mathrm{M}$ can be derived in $\mathrm{E}^{\otimes}$ plus $\otimes-\mathrm{RM}$.
1. $\bigwedge_{i=1}^{n}\left(a_{i} \wedge b_{i}\right) \rightarrow b_{i}$
Tautology
2. $\bigotimes_{i=1}^{n}\left(a_{i} \wedge b_{i}\right) \rightarrow \bigotimes_{i=1}^{n} b_{i}$
1., $\otimes-\mathrm{RM}$
3. $\bigwedge_{i=1}^{n}\left(a_{i} \wedge b_{i}\right) \rightarrow a_{i}$
Tautology
4. $\bigotimes_{i=1}^{n}\left(a_{i} \wedge b_{i}\right) \rightarrow \bigotimes_{i=1}^{n} a_{i}$
3., $\otimes-\mathrm{RM}$
5. $\bigotimes_{i=1}^{n}\left(a_{i} \wedge b_{i}\right) \rightarrow\left(\bigotimes_{i=1}^{n} a_{i} \wedge \bigotimes_{i=1}^{n} b_{i}\right)$
2., 4., PC

\subsection{Expansion}

The formulation of expansion is also quite intuitive. Consider the following example.

Example 7.6. Let $G=\{$ Erica, Serena, Anna $\}$ be the group of girls in a class $T=G \cup B$ consisting of boys and girls and where $B=\{$ Guido $\}$. If I prefer Erica and Serena (they are for me the best elements) as the fastest runners in the $100 \mathrm{~m}$ (as part of $G$ ), then Erica and Serena are the best elements as they are among the fastest runners in the whole class, or none of them is among the fastest ones.

Pairs of alternatives (more generally, pairs of sets of alternatives) can be selected by assuming the truth of pairs of formulae in $\otimes$-chains. Now, if we pick up larger sets (which are determined by disjunctively adding any arbitrary propositional formula), then either the same choice is preserved, or it is abandoned in both alternatives. Formally, we can represent the example as follows:

$$
\begin{gathered}
(\text { SerenaFastest-G } \otimes \text { AnnaFastest-G }) \wedge(\text { EricaFastest-G } \otimes \text { AnnaFastest-G }) \rightarrow \\
\rightarrow(((\text { SerenaFastest-G } \vee \text { SerenaFastest-B }) \otimes \text { AnnaFastest-G })) \equiv \\
\equiv(((\text { EricaFastest-G } \vee \text { EricaFastest-B }) \otimes \text { AnnaFastest-G }))
\end{gathered}
$$

Accordingly, expansion can be in general captured by the following axiom schema: 


$$
\begin{aligned}
\left(\bigotimes_{i=0}^{m} a_{i} \otimes a \otimes \bigotimes_{j=0}^{n} d_{j} \wedge \bigotimes_{i=0}^{m} a_{i} \otimes b \otimes \bigotimes_{j=0}^{n} d_{j}\right) & \rightarrow \\
& \left(\left(\bigotimes_{i=0}^{m} a_{i} \otimes(a \vee c) \otimes \bigotimes_{j=0}^{n} d_{j}\right) \equiv\left(\bigotimes_{i=0}^{m} a_{i} \otimes(b \vee c) \otimes \bigotimes_{j=0}^{n} d_{j}\right)\right)
\end{aligned}
$$

$(\otimes$-expansion)

Expansion is characterised by the following frame property.

Definition 7.7. Let $\mathcal{F}=\langle W, \mathcal{C}\rangle$ be a sequence frame. We say that $\mathcal{F}$ is $\otimes$-expanded iff, for any $w \in W$, if $\left\langle X_{0}, \ldots, X_{m}, Y, Z_{0}, \ldots, Z_{n}\right\rangle \in \mathcal{C}_{w}$ and $\left\langle X_{0}, \ldots, X_{m}, W, Z_{0}, \ldots, Z_{n}\right\rangle \in \mathcal{C}_{w}$, then $\left\langle X_{0}, \ldots, X_{m}, Y \cup\right.$ $\left.P, Z_{0}, \ldots, Z_{n}\right\rangle \in \mathcal{C}_{w}$ iff $\left\langle X_{0}, \ldots, X_{m}, W \cup P, Z_{0}, \ldots, Z_{n}\right\rangle \in \mathcal{C}_{w}$.

Lemma 7.8. ( $\otimes$-expansion) is valid in the class of $\otimes$-expanded sequence frames.

\section{Proof:}

Suppose by reductio that there is an $\otimes$-expanded frame $\mathcal{F}$, a valuation $V$, and a world $v$ such that

$$
\begin{gathered}
\models_{v}^{V}\left(\bigotimes_{i=0}^{m} a_{i} \otimes a \otimes \bigotimes_{j=0}^{n} d_{j}\right) \wedge\left(\bigotimes_{i=0}^{m} a_{i} \otimes b \otimes \bigotimes_{j=0}^{n} d_{j}\right) \\
\forall_{v}^{V}\left(\left(\bigotimes_{i=0}^{m} a_{i} \otimes(a \vee c) \otimes \bigotimes_{j=0}^{n} d_{j}\right) \equiv\left(\bigotimes_{i=0}^{m} a_{i} \otimes(b \vee c) \otimes \bigotimes_{j=0}^{n} d_{j}\right)\right)
\end{gathered}
$$

From (9) we obtain that there are two sequences $\left\langle\left\|a_{0}\right\|_{V}, \ldots,\left\|a_{m}\right\|_{V},\|a\|_{V},\left\|d_{0}\right\|_{V}, \ldots,\left\|d_{n}\right\|_{V}\right\rangle \in$ $\mathcal{C}_{v}$ and $\left\langle\left\|a_{0}\right\|_{V}, \ldots,\left\|a_{m}\right\|_{V},\|b\|_{V},\left\|d_{0}\right\|_{V}, \ldots,\left\|d_{n}\right\|_{V}\right\rangle \in \mathcal{C}_{v}$. From (10) we have that there is no sequence $\left\langle\left\|a_{0}\right\|_{V}, \ldots,\left\|a_{m}\right\|_{V},\|a\|_{V} \cup\|c\|_{V},\left\|d_{0}\right\|_{V}, \ldots,\left\|d_{n}\right\|_{V}\right\rangle \in \mathcal{C}_{v}$ while there a sequence $\left\langle\left\|a_{0}\right\|_{V}, \ldots,\left\|a_{m}\right\|_{V},\|b\|_{V} \cup\|c\|_{V},\left\|d_{0}\right\|_{V}, \ldots,\left\|d_{n}\right\|_{V}\right\rangle \in \mathcal{C}_{v}$; thus, we have that $\mathcal{F}$ is not $\otimes$-expanded, contrary to our assumption.

Notice that $\otimes$-expansion is canonical, thus completeness is guaranteed.

\section{Theorem 7.9. (Completeness of $E^{\otimes}$ plus $\otimes$-expansion)}

$E^{\otimes}$ plus $\otimes$-expansion is complete with respect to the class of sequence frames that are $\otimes$-expanded.

\section{Proof:}

Let us consider a canonical model for ( $\otimes$-expansion) and any two sequences

$$
\begin{aligned}
& \left\langle\left\|a_{0}\right\|_{V}, \ldots,\left\|a_{m}\right\|_{V},\|a\|_{V},\left\|d_{0}\right\|_{V}, \ldots,\left\|d_{n}\right\|_{V}\right\rangle \in \mathcal{C}_{w} \\
& \left\langle\left\|a_{0}\right\|_{V}, \ldots,\left\|a_{m}\right\|_{V},\|b\|_{V},\left\|d_{0}\right\|_{V}, \ldots,\left\|d_{n}\right\|_{V}\right\rangle \in \mathcal{C}_{w} .
\end{aligned}
$$

Clearly, $\left(\bigotimes_{i=0}^{m} a_{i} \otimes a \otimes \bigotimes_{j=0}^{n} d_{j}\right) \in w$ and $\left(\bigotimes_{i=0}^{m} a_{i} \otimes b \otimes \bigotimes_{j=0}^{n} d_{j}\right) \in w$, thus $\left(\bigotimes_{i=0}^{m} a_{i} \otimes a \otimes \bigotimes_{j=0}^{n} d_{j}\right) \wedge$ $\left(\bigotimes_{i=0}^{m} a_{i} \otimes b \otimes \bigotimes_{j=0}^{n} d_{j}\right) \in w$. Since $\left(\otimes\right.$-expansion) is valid (Lemma 7.8), then $\left(\left(\bigotimes_{i=0}^{m} a_{i} \otimes(a \vee c) \otimes\right.\right.$ $\left.\bigotimes_{j=0}^{n} d_{j}\right) \equiv\left(\bigotimes_{i=0}^{m} a_{i} \otimes(b \vee c) \otimes \bigotimes_{j=0}^{n} d_{j}\right) \in w$. Hence, $\left(\left(\bigotimes_{i=0}^{m} a_{i} \otimes(a \vee c) \otimes \bigotimes_{j=0}^{n} d_{j}\right)\right.$ iff $\left(\bigotimes_{i=0}^{m} a_{i} \otimes\right.$ $\left.(b \vee c) \otimes \bigotimes_{j=0}^{n} d_{j}\right) \in w$. By construction, $\left\langle\left\|a_{0}\right\|_{V}, \ldots,\left\|a_{m}\right\|_{V},\|a\|_{V} \cup\|c\|_{V},\left\|d_{0}\right\|_{V}, \ldots,\left\|d_{n}\right\|_{V}\right\rangle \in \mathcal{C}_{w}$ iff $\left\langle\left\|a_{0}\right\|_{V}, \ldots,\left\|a_{m}\right\|_{V},\|b\|_{V} \cup\|c\|_{V},\left\|d_{0}\right\|_{V}, \ldots,\left\|d_{n}\right\|_{V}\right\rangle \in \mathcal{C}_{w}$. Therefore, the model is $\otimes$-expanded. 


\section{Conclusion and Related Work}

This paper offered a semantic study of the $\otimes$ operator originally introduced in [9] to model deontic reasoning and contrary-to-duty obligations. We showed that a suitable axiomatisation incorporating $\otimes$ expressions can be characterised in a class of structures extending neighbourhood frames with sequences of sets of worlds. We argued that both the formalism, and the semantics can be employed, with some adjustments, to grasp various forms of reasoning about reason-based preferences. In this perspective, our contribution may offer useful insights for establishing connections between the proof-theoretic and model theoretic approaches to preference reasoning. Also, we presented a logic that validates both Contraction and Expansion Consistency [1,6], thus satisfying two basic rationality conditions in social choice theory.

Our system falls within the research on prioritised goals [4, 5], i.e., on formalisms for describing the goals of the agents whose preferences are modelled as propositional formulae. This allows for a purely qualitative representation of preferences. Before the recent developments in MAS [2], the most extensive (and, still the most advanced) work on preferences was done in the context of deontic logic. A first line of inquiry was mainly semantic-based: deontic sentences are interpreted in settings with ideality orderings on possible worlds or states [10]. This approach is quite flexible: depending on the properties of the preference or ideality relation, different deontic logics can be obtained. This semantic approach has been fruitfully renewed in the ' 90 for example by $[18,19]$, and most recently by works such as [11, 12], which have confirmed the vitality of this line of inquiry. The second line was proof-theoretic: in this second area, the Gentzen system proposed in [9] was definitely seminal for us in developing the current proposal. [9] is based on the introduction of the non-classical binary operator $\otimes$ : The reading of an expression like $a \otimes b$ is that $a$ is primarily obligatory, but if the obligation is violated, the secondary obligation is $b$. Inference rules introduced by $[9]$ —in particular, $(\otimes$-shortening $)$ and $(\otimes-\mathrm{I})$ —are proposed here, too.

In the context of preference logics, several proposals can be mentioned [4, 5, 7]. However, two works have specifically inspired our effort: [6] and [8]. In the recent [6], the authors present a modal logic where a binary operator $\nabla$ is meant to syntactically express preference orderings between formulae: Each formula of this logic determines a preference ordering over alternatives based on the priorities over properties that the formula express. While the formalism is interesting in that it can represent not just orderings over alternatives, but also the reasons that lead to such preferences [7], the modal logic for expressing individual preferences is in fact equivalent to $\mathrm{S} 5$; this can be easily illustrated by inspecting the semantic conditions for evaluating $\nabla$-expressions: Given the set $W$ of worlds and $w \in W, \models_{w}^{V} a \nabla b$ iff $\models_{w}^{V} a$, or $\models_{w}^{V} b$ and $\nvdash_{w^{\prime}}^{V} a$ for all $w^{\prime} \in W$. In other words, the logic of [6] amounts to being a very strong and simple option (indeed, the main concern in this work is preference aggregation): As we argued, weaker, though very expressive logics can be adopted. The qualitative choice logic (QCL) of [8] is a propositional logic for representing alternative, ranked options for problem solutions, using a substructural ordered disjunction $\vec{x}$. It offers a much richer alternative with respect to [6], showing a number of similarities with [9] (the two formalisms have been developed independently) and the one discussed here. For example, the detachment rule from $\otimes$-chain is exactly the same. The semantics and proof theory of [8], though based on similar intuitions, are however technically different from ours: Semantics is based on the degree of satisfaction of a formula in a particular classical model. More precisely, if an interpretation $I$ is an assignment of the classical truth values true and false to the atoms, the satisfaction degree $k$ of $a_{1} \overrightarrow{\times} \ldots \overrightarrow{\times} a_{n}$ in $I$ is simply the smallest $k$ such that $I$ satisfies $a_{k}$. If none of the ordered disjuncts is satisfied, then also the formula is not satisfied. Consequences of QCL theories can be computed through a compilation to stratified knowledge bases which in turn can be compiled to classical 
propositional theories. The consequence relation of [8] satisfies properties usually considered intended in nonmonotonic reasoning, such as cautious monotonicity and cumulative transitivity. Our approach is by contrast monotonic, as it is developed in a modal-logic setting as a direct extension of classical modal system E [15]. Another difference is that in our approach we can speak about what an agent prefers using the Properator. This allows us to express preferences conditional to what the agent actually prefers in a specific situation, for example $\operatorname{Pr} a \rightarrow b \otimes c$, meaning that if $a$ is one of the preferred outcomes of the agent, then the agent prefers $b$ to $c$.

A number of open research issues are left for future work. The logic of $\otimes$ proved to be flexible for several applied domains. For example [20] shows how different conditions on the operator can be used for reasoning about different types of defeasible permission. Similarly, [21] investigates how to characterise different degrees and types of goal-like mental attitudes of agents. These works assume defeasible logic as the underlying logic and they are restricted to literals. However, they show that the extension of defeasible logic obtained from adding $\otimes$ are still computationally feasible. The natural question is to see how to use the sequence semantics we have presented in this paper to capture the different intuitions of $\otimes$ discussed in the above mentioned work. In addition we plan to explore decidability questions using, for example, the filtration methods. The fact that neighbourhoods contain sequences of sets of worlds instead of sets is not expected to make the task significantly harder than the one in standard neighbourhood semantics for modal logics.

Second, we expect to enrich the language and allow for nesting of $\otimes$-expressions, thus having formulae like $a \otimes \neg(b \otimes c) \otimes d$. We argued in [9] that the meaning of such formulae is not clear in deontic reasoning. However, a semantic analysis of them within the sequence semantics can clarify the issue. Indeed, in the current language we can evaluate in any world $w$ a formula such as $\neg(a \otimes b)$, which semantically means that there is no sequence $\left\langle\|a\|_{V},\|b\|_{V}\right\rangle \in \mathcal{C}_{w}$. Conceptually, expressions of this type may express meta-preferences, i.e., preferences about preference orderings. However, this reading poses interesting conceptual as well as technical problems.

Finally, we plan to apply our framework to social choice theory by checking how our analysis impacts on the collective choice rules proposed in [6]. In particular, since a collective choice rule is a function $f$ that assigns to each profile of individual choices (i.e., a complete ranking covering all agents) a single formula expressing non-individual preferences, we expect to preliminarily offer proof- and model-theoretic characterisations of the following principles [6, p. 982].

Unrestricted domain: The domain of the collective choice rule $f$ includes all profiles of individual choices.

Anonymity: The ordering among the agents does not affect the collective result and the collective rule should treat each individual neutrally.

Monotonicity: for any two preference profiles and any alternative if for each individual the ranking of the alternative in one profile is at least as high as that in the other, then its collective ranking of the former is at least as high as that of the latter.

Pareto principle: if all individual priorities over reasons are the same, then this condition requires that the collective priority over reasons is the same as each individual's one. 


\section{Acknowledgements}

This paper is the extended and revised version of [22] presented at the 18th International Conference on Principles and Practice of Multi-Agent Systems (PRIMA 2015), Bertinoro, Italy, 26-30 October 2015.

The research was supported by the European Union's Horizon 2020 research and innovation programme under the Marie Skłodowska-Curie grant agreement No 690974 for the project MIREL: MIning and REasoning with Legal texts.

\section{References}

[1] Gaertner W. A Primer in Social Choice Theory: Revised Edition. Oxford University Press; 2009.

[2] Brandt F, Conitzer V, Endriss U. Computational Social Choice. In: Multiagent Systems. MIT Press; 2012. .

[3] Boutilier C, Brafman RI, Domshlak C, Hoos HH, Poole D. CP-nets: A Tool for Representing and Reasoning with Conditional Ceteris Paribus Preference Statements. Journal of Artificial Intelligence Research. 2004;21:135-191. doi:10.1613/jair.1234.

[4] Coste-Marquis S, Lang J, Liberatore P, Marquis P. Expressive Power and Succinctness of Propositional Languages for Preference Representation. In: Principles of Knowledge Representation and Reasoning: Proceedings of the Ninth International Conference (KR 2004); 2004. p. 203-212. Available from: http: //www . aaai.org/Library/KR/2004/kr04-023.php.

[5] Lang J. Logical Preference Representation and Combinatorial Vote. Annals of Mathematics in Artificial Intelligence. 2004;42(1-3):37-71. doi:10.1023/B:AMAI.0000034522.25580.09.

[6] Jiang G, Zhang D, Perrussel L, Zhang H. A Logic for Collective Choice. In: Proceedings of the 2015 International Conference on Autonomous Agents and Multiagent Systems (AAMAS 2015); 2015. p. 979-987. Available from: http://dl. acm.org/citation. cfm?id=2773277.

[7] Osherson D, Weinstein S. Preference based on reasons. The Review of Symbolic Logic. 2012 3;5:122-147. doi:10.1017/S1755020311000244.

[8] Brewka G, Benferhat S, Le Berre D. Qualitative choice logic. Artificial Intelligence. 2004;157(1-2):203-237. doi:10.1016/j.artint.2004.04.006.

[9] Governatori G, Rotolo A. Logic of Violations: A Gentzen System for Reasoning with Contrary-To-Duty Obligations. Australasian Journal of Logic. 2006;4:193-215.

[10] Hansson B. An Analysis of Some Deontic Logics. Nous. 1969;(3):373-398.

[11] Hansen J. Conflicting imperatives and dyadic deontic logic. Journal of Applied Logic. 2005;3(3-4):484-511.

[12] van Benthem J, Grossi D, Liu F. Priority Structures in Deontic Logic. Theoria. 2014;80(2):116-152. doi:10.1111/theo.12028.

[13] Calardo E, Governatori G, Rotolo A. A Preference-Based Semantics for CTD Reasoning. In: Deontic Logic and Normative Systems - 12th International Conference (DEON 2014); 2014. p. 49-64.

[14] Governatori G, Olivieri F, Calardo E, Rotolo A. A Sequence Semantics for Norms and Obligations. In: Deontic Logic and Normative Systems - 13th International Conference (DEON 2016). College Publications; 2016. p. 93-108.

[15] Chellas BF. Modal Logic, An Introduction. Cambridge University Press; 1980. 
[16] Governatori G. Thou Shalt is not You Will. In: Atkinson K, editor. Proceedings of the Fifteenth International Conference on Artificial Intelligence and Law. New York: ACM; 2015. p. 63-68. doi:10.1145/2746090.2746105.

[17] Sen A. Social Choice Theory: A Re-examination. Econometrica. 1977;45(1):53-89. Available from: http://EconPapers.repec.org/RePEc : ecm:emetrp:v:45:y:1977:i:1:p:53-89.

[18] Prakken H, Sergot MJ. Contrary-to-Duty Obligations. Studia Logica. 1996;57(1):91-115.

[19] van der Torre L. Reasoning about obligations: defeasibility in preference-based deontic logic. PhD thesis, Erasmus University Rotterdam; 1997.

[20] Governatori G, Olivieri F, Rotolo A, Scannapieco S. Computing Strong and Weak Permissions in Defeasible Logic. Journal of Philosophical Logic. 2013;42(6):799-829.

[21] Governatori G, Olivieri F, Scannapieco S, Rotolo A, Cristani M. The Rational behind the Concept of Goal. Theory and Practice of Logic Programming. 2016;16:296-324. doi:10.1017/S1471068416000053.

[22] Calardo E, Governatori G, Rotolo A. Semantics for Modelling Reason-based Preferences. In: Chen Q, Torroni P, Villata S, editors. Proceedings PRIMA 2015. vol. 9387 of LNCS. Springer; 2015. p. 101-117. doi:10.1007/978-3-319-25524-8 7. 